\title{
A tentative replica study of the glass transition
}

\author{
Marc Mézard ${ }^{1}$ and Giorgio Parisi ${ }^{2}$ \\ ${ }^{1}$ Laboratoire de Physique Théorique de l'Ecole Normale Supérieure*, \\ 24 rue Lhomond, 75231 Paris Cedex 05, France \\ ${ }^{2}$ Dipartimento di Fisica, Università La Sapienza \\ INFN Sezione di Roma I \\ Piazzale Aldo Moro, Roma 00187, Italy
}

\begin{abstract}
In this paper we propose a method to study quantitatively the glass transition in a system of interacting particles. In spite of the absence of any quenched disorder, we introduce a replicated version of the hypernetted chain equations. The solution of these equations, for hard or soft spheres, signals a transition to the glass phase. However the predicted value of the energy and specific heat in the glass phase are wrong, calling for an improvement of this method.
\end{abstract}

LPTENS preprint 96/12.

Electronic addresses : mezard@physique.ens.fr, parisi@roma1.infn.it

*Unité propre du CNRS, associée à l'Ecole Normale Supérieure et à l'Université de Paris Sud 


\section{Introduction}

The long lasting studies of the glass transition, in spite of recent progress [1], have not yet produced a completely convincing theory deriving the low temperature behavior from a microscopic Hamiltonian of interacting particles.

It has been proposed that near this transition real glasses could behave in the same way as some disordered systems which are described by a Hamiltonian with quenched randomness [2, 3, 4, 5, 6]. This proposal is supported the existence of a wide class of mean field systems, with fixed non random Hamiltonians which show a typical glassy behaviour 17, 8, 5, 9, 10, 11, 12, 13. Although they depend on one or a few coupling constants, these systems behave as if they were typical samples of a class of systems with quenched randomness (apart from the possible existence of a crystal phase 8 ).

The relevant disordered systems found so far have the following behaviour at the mean field level. There are two phase transitions: a usual static transition at low temperature $\left(T_{R}\right)$, where the replica symmetry is broken and the specific heat is discontinuous, and a dynamic transition at a higher temperature $\left(T_{D}\right)$, where some time persistent correlations set in. In the region where $T<T_{D}$ the infinite system, quenched from some high energy configuration, gets trapped into metastable states, such that its energy density is larger than the equilibrium value. In this dynamics the metastability appears at $T_{R}$ and the thermodynamic transition, which is present at a lower temperature, is not accessible [2, 14, 15, 16, 18. Beyond the mean field approximation one finds that the equilibration time is not divergent at $T_{D}$, but it becomes exponentially large in the region $T_{C}<T<T_{D}$. It has been argued that in some spin model the equilibration time diverges (as $\exp \left(A\left|T-T_{C}\right|^{-\gamma}\right.$ ), $\gamma=d-1$ in $d$ dimensions [3, 1 . This problem will not be studied here, we shall stay at a mean field level.

In this paper we want to present a progress report on an analytical study of the glass transition for a real three dimensional model of interacting particles.

\section{Breaking the replica symmetry}

We consider a system of $N$ interacting particle in a volume $V$. We study the infinite volume limit in which $N \rightarrow \infty$ at fixed $\rho \equiv N / V$. The Hamiltonian is given by:

$$
H(x)=\sum_{i<k} U\left(x_{i}-x_{k}\right)
$$

We will consider two forms of the potential among particles: The hard sphere case where $U(x)=\infty$ for $r \equiv|x|<1$ and $U(x)=0$ for $r>1$, and a soft sphere case $U(x)=r^{-12}$.

In both cases, if the system is cooled from high to low temperature fast enough, crystalization is inhibited and the system undergoes a glass transition. For hard spheres this transition occurs at a density of about 1.15 independently from the temperature, while the crystallisation would occur for an equilibrium system at density around .95. In the

case of soft spheres thermodynamic quantities are functions of the dimensionless density $\gamma \equiv \rho T^{(-1 / 4)}$ and the glass transition is located around $\gamma \simeq 1.6$, while the freezing value of $\gamma$ is around $\gamma \simeq 1.15$ [19].

It is possible to consider $n$ replicas of the same system and to study the total partition 
function

$$
Z=\int \prod_{i, a} d x_{i}^{a} \exp -\beta\left(\sum_{a} H\left(x^{a}\right)+\epsilon \sum_{a, b} \sum_{i \neq k} \phi_{a, b}\left(x_{i}^{a}-x_{k}^{b}\right)\right) .
$$

The detailed form of the (attractive) potential $\phi_{a, b}$ is not relevant here because we will eventually set $\epsilon=0$.

Replica symmetry breaking corresponds to the case in which (in the infinite system) the correlation among replicas does not vanish in the limit $\epsilon \rightarrow 0$. The physical meaning of this breaking is clear: at high density, if several metastable states contribute to the Boltzmann measure, different replicas may be frozen in the same state. Indeed we can propose the following picture of the glass phase.

There are many equilibrium states, which we label by an index $\alpha$ (modulo rotations and translations). These states are identified by the density $<\hat{\rho}(x)>_{\alpha} \equiv \rho_{\alpha}(x)$ which depends on $x$ in each state. In this paper we denote by $\hat{\rho}(x)$ the density operator $\sum_{j} \delta\left(x-x_{j}\right)$ and similarly by $\hat{\rho}_{a}(x)$ the density operator in replica $a$. The brackets stand for thermal expectation values. Each state $\alpha$ appears in the Boltzmann measure with a weight $w_{\alpha}$. We assume that there are many states, and the quantities which we can access are averaged over the states. Since the one point average $\sum_{\alpha} w_{\alpha} \rho_{\alpha}(x)=\rho$ is uniform, we need to study the two point correlations like:

$$
\begin{aligned}
\tilde{g}(x, y) & =\frac{1}{\rho^{2}} \sum_{\alpha} w_{\alpha} \sum_{i \neq j}<\delta\left(x_{i}-x\right) \delta\left(x_{j}-y\right)>_{\alpha} \\
g_{1}(x, y) & =\frac{1}{\rho^{2} \sum_{\alpha} w_{\alpha}^{2}} \sum_{\alpha} w_{\alpha}^{2} \sum_{i \neq j}<\delta\left(x_{i}-x\right)>_{\alpha}<\delta\left(x_{j}-y\right)>_{\alpha}
\end{aligned}
$$

As for the densities in two different states, $\alpha \neq \beta$, we assume that they are uncorrelated.

In replica space one could thus expect the correlations to be, for $a \neq b:\left\langle\hat{\rho}_{a}(x) \hat{\rho}_{b}(y)\right\rangle=$ $w_{2} \rho_{\alpha}(x) \rho_{\alpha}(y)=w_{2} \rho^{2} g_{1}(x, y)$, where we use the notation

$$
w_{k}=<\sum_{\alpha} w_{\alpha}^{k}>=\int d w P(w) w^{k} .
$$

Notice that we are describing the quantities $w$ by their probability distribution $P(w)$. This description may look strange for such a deterministic problem (the Hamiltonian is not random). It is clear that in a finite volume, at fixed $N$, the $w$ 's are given numbers. However they may strongly depend on $N$ so that if we average over a small window in $N$ around $\bar{N}$ and send $\bar{N} \rightarrow \infty$ we induce a smooth probability distribution for these variables. In this formulation one finds the unpleasant feature that the correlation function of more replicas do not factorize when the distance goes infinity. For instance, the four point function

$$
<\hat{\rho}_{a}(x) \hat{\rho}_{b}(y) \hat{\rho}_{c}(z) \hat{\rho}_{d}(t)>=\sum_{\alpha} w_{\alpha}^{4} \rho_{\alpha}(x) \rho_{\alpha}(y) \rho_{\alpha}(z) \rho_{\alpha}(t)
$$

goes to $w_{4} g_{1}(x, y) g_{1}(z, t)$ when $x-y=O(1), z-t=O(1)$ and $x-z \rightarrow \infty$, which is different from the factorized form $\left(w_{2}\right)^{2} g_{1}(x, y) g_{1}(z, t)$.

The non factorization of the correlation function is deeply linked to the probability distribution of the variables $w$. This crucial ingredient of the description is usually dealt with by using the replica symmetry breaking formalism. This means that the correlation functions (e.g. $<\hat{\rho}_{a}(x) \hat{\rho}_{b}(y)>$ ) are no more symmetric under the permutation of the 
replicas, but they have the advantage of being clustering 円. This formalism provides and automatic bookkeeping of all complications which would arise from the existence of many states. If one assumes a given form for replica symmetry breaking, it corresponds to a given form for the probability distribution of the $w$. In the simplest case, called one step breaking, one divides the $n$ replicas in $n / m$ groups of $m$ replicas and one assumes the following structure of correlation functions:

$$
\begin{aligned}
& <\hat{\rho}_{a}(x) \hat{\rho}_{a}(y)>=\rho^{2} \tilde{g}(x-y) \\
& <\hat{\rho}_{a}(x) \hat{\rho}_{b}(y)>=\rho^{2} g_{1}(x-y) \text { for } a \neq b \text { in the same group } \\
& <\hat{\rho}_{a}(x) \hat{\rho}_{b}(y)>=\rho^{2} \text { for } a \text { and } b \text { in different groups. }
\end{aligned}
$$

In the physically relevant case where $m<1$, this form of the correlations corresponds to assume that $w_{\alpha}=\exp \left(-\beta f_{\alpha}\right) /\left[\sum_{\alpha^{\prime}} \exp \left(-\beta f_{\alpha^{\prime}}\right)\right]$ where the $f_{\alpha}$ are negative quantities extracted with a probability distribution $\beta m \exp (\beta m f)$. The details of this construction are described extensively in the literature [20].

May be the reader has some familiarity with the replica formalism and he is waiting here for the canonical sentence we send $n$ to zero at the end of the computation. However this is not a random system and there is nothing to average (apart from the value of $N$ ), there is no need to send $n$ to zero. Indeed it turns out that in this scheme, the free energy density, $-\ln (Z) /(\beta N n)$, is independent of $n$ as soon as $<\hat{\rho}_{a}(x) \hat{\rho}_{b}(y)>-<\hat{\rho}_{a}(x)><\hat{\rho}_{b}(y)>=0$ for $a$ and $b$ in different groups. In some sense $n$ is infinite, because we assume that $m$ divides $n$ for any $m$.

In this scheme the free energy depends on $m$, which should be considered as a variational parameter and the free energy must be maximized (not minimized!) as usual in the replica approach. For $m=1$ we recover the replica symmetric result. The static glass transition temperature $T_{R}$ is characterized by the existence of a maximum of the free energy at $m(T)<1$, where $m\left(T_{R}\right)=1$. On the other hand, the appearance of metastable states which will trap the dynamical evolution of the system is signalled by the existence of a non trivial $g_{1}(x)$ in the limit $m \rightarrow 1$ [16, 17].

The programme is thus clear. The real difficulty consists now in implementing it, i.e. in computing the properties of our replicated system of interacting particles, allowing for replica symmetry breaking. A first step in this direction will be proposed in the next section.

\section{The replicated hypernetted chain approximation}

The hypernetted chain (HNC) approximation consists in considering only a given class of diagrams in the virial expansion [21]. It gives a reasonable account of the liquid phase, and it has also been used for studying the first order transition to the crystal phase [22]. We will consider here this approximation because it has the advantage of having a simple variational formulation. In the liquid phase, where the density is constant, the usual HNC equation (for the non replicated system) can be written as

$$
g(x)=\exp (-\beta U(x)+W(x))
$$

\footnotetext{
${ }^{1}$ We thank Francesco Guerra for discussions on this point.
} 
where:

$$
\begin{aligned}
\rho^{2} g(x) & =\rho^{2}(1+h(x))=<\hat{\rho}(x) \hat{\rho}(0)>-\rho \delta(x), \\
W(x) & \equiv \int \frac{d^{d} p}{(2 \pi)^{d}} e^{-i p x} \frac{\rho \mathbf{h}(p)^{2}}{1+\rho \mathbf{h}(p)},
\end{aligned}
$$

and we denote by $\mathbf{h}(p)$ the Fourier transform of $h(x)$.

This equation can be derived by minimizing with respect to $h(x)$ the following free energy per unit volume, in the space of functions of $|x|$ :

$$
2 \beta F=\int d^{d} x \rho^{2} g(x)[\ln (g(x))-1+\beta U(x)]+\int \frac{d^{d} q}{(2 \pi)^{d}} L(\rho \mathbf{h}(q)),
$$

where $L(x) \equiv-\ln (1+x)+x-x^{2} / 2$.

In this note we propose a bold generalization of the HNC equations for $n$ replicas. The replicated free energy is now

$$
2 \beta F=\rho^{2} \int d^{d} x \sum_{a, b} g_{a b}(x)\left[\ln \left(g_{a b}(x)\right)-1-\beta\left(U(x) \delta_{a, b}+\epsilon \phi_{a b}\right)\right]+\operatorname{Tr} L(\rho \mathbf{h}),
$$

where $\mathbf{h}$ is now an operator both in $x$ space and in replica space.

If in the limit $\epsilon \rightarrow 0$ one finds that at large enough densities $g_{a b}$ is non zero off the diagonal, replica symmetry is broken. In the case where $g_{a b}$ is of the form shown in eq. (6), this equation can be used to compute the properties of the correlation function in the glassy phase. This approach amounts to a study of the density modulations in the glass phase at the level of the two point function. In the glass phase $\rho_{\alpha}(x)$ becomes space dependent. However, as argued in the introduction, the necessity of averaging over the states $\alpha$ forces us to study this $x$ dependence at the level of the two point correlations: We keep here to the definition of $\tilde{g}$ through $\sum_{\alpha} w_{\alpha}<\hat{\rho}(x) \hat{\rho}(y)>_{\alpha}=\delta(x-y) \sum_{\alpha} w_{\alpha} \rho_{\alpha}(x)+\rho^{2} \tilde{g}(x, y)$, while the second term should be written more properly as $\rho_{\alpha}(x) g_{\alpha}(x, y) \rho_{\alpha}(y)$. So our correlation $\tilde{g}$ reflects the structure of $\rho_{\alpha}(x)$ as a sum of peaks of unit weights, smoothed by the average over states.

Within the one step breaking scheme (6), the free energy is:

$$
\begin{array}{r}
\lim _{n \rightarrow 0} \frac{2 \beta F}{n \rho^{2}}=\int d^{d} x\{\tilde{g}(x)[\ln (\tilde{g}(x)) \\
\left.-1+\beta U(x)]-(1-m) g_{1}(x)\left[\ln \left(g_{1}(x)\right)-1\right]\right\} \\
-\int \frac{d^{d} q}{(2 \pi)^{d}}\left(\frac{\tilde{h}(q)^{2}}{2}-(1-m) \frac{h_{1}(q)^{2}}{2}-\frac{\tilde{h}(q)}{\rho}\right. \\
\left.+\frac{1}{m \rho^{2}} \ln \left[1+\rho \tilde{h}(q)-(1-m) \rho h_{1}(q)\right]-\frac{1-m}{m \rho^{2}} \ln \left[1+\rho \tilde{h}(q)-\rho h_{1}(q)\right]\right)
\end{array}
$$

The static transition is identified as the temperature (or density) at which there exists a non trivial solution to the replicated HNC equations $\partial F / \partial \tilde{g}(x)=0, \partial F / \partial g_{1}(x)=0$ and $\partial F / \partial m=0$, for $m \in[0,1]$ (in fact we must minimize the free energy with respect to $\tilde{g}$, but maximize with respect to $g_{1}$ and $m$ ). The equations to be solved for the statics are thus:

$$
\begin{array}{r}
\tilde{g}(x)=\exp (-\beta U(x)+\tilde{W}(x)), \quad g_{1}(x)=\exp \left(W_{1}(x)\right) \\
\int d^{d} x g_{1}(x)\left[\ln \left(g_{1}(x)\right)-1\right]=\int \frac{d^{d} q}{(2 \pi)^{d}}\left(\frac{\mathbf{h}_{\mathbf{1}}(q)^{2}}{2}+\frac{1}{m \rho} \frac{\mathbf{h}_{\mathbf{1}}(q)}{1+\rho \tilde{\mathbf{h}}(q)-(1-m) \rho \mathbf{h}_{\mathbf{1}}(q)}\right. \\
\left.-\frac{1}{m^{2} \rho^{2}} \ln \frac{1+\rho \tilde{\mathbf{h}}(q)-(1-m) \rho \mathbf{h}_{\mathbf{1}}(q)}{1+\rho \tilde{\mathbf{h}}(q)-\rho \mathbf{h}_{\mathbf{1}}(q)}\right)
\end{array}
$$


where the Fourier transforms $\tilde{\mathbf{W}}(q)$ and $\mathbf{W}_{\mathbf{1}}(q)$ of $\tilde{W}(x)$ and $W_{1}(x)$ satisfy:

$$
\begin{aligned}
\tilde{\mathbf{W}}(q)-(1-m) \mathbf{W}_{\mathbf{1}}(q) & =\rho \frac{\left(\tilde{\mathbf{h}}(q)-(1-m) \mathbf{h}_{\mathbf{1}}(q)\right)^{2}}{1+\rho\left(\tilde{\mathbf{h}}(q)-(1-m) \mathbf{h}_{\mathbf{1}}(q)\right)} \\
\mathbf{W}_{\mathbf{1}}(q) & =\frac{1}{m}\left(\frac{\rho\left(\tilde{\mathbf{h}}(q)-(1-m) \mathbf{h}_{\mathbf{1}}(q)\right)^{2}}{1+\rho\left(\tilde{\mathbf{h}}(q)-(1-m) \mathbf{h}_{\mathbf{1}}(q)\right)}-\frac{\rho\left(\tilde{\mathbf{h}}(q)-\mathbf{h}_{\mathbf{1}}(q)\right)^{2}}{1+\rho\left(\tilde{\mathbf{h}}(q)-\mathbf{h}_{\mathbf{1}}(q)\right)}\right)(14)
\end{aligned}
$$

The dynamical transition may be characterized as the highest temperature at which there is a non trivial solution of the stationarity equations $\partial F / \partial \tilde{g}(x)=0$ and $\partial F / \partial g_{1}(x)=$ 0 at $m=1^{-}$. The corresponding equations are obtained by substituting $m \rightarrow 1$ in the first two equations of (13). The equation for $\tilde{g}$ is identical to the usual HNC equation (8), while $g_{1}$ is a solution of $g_{1}(x)=\exp \left(W_{1}(x)\right)$, with $W_{1}$ given by the second equation of (14) at $m=1$.

Within this static approach there is no obvious definition of the dynamic energy. Previous work [16, 17] suggests the following computation: one considers a system at equilibrium at a temperature $T_{R}>T_{G}$ and an other system at temperature $T$. One defines the correlation functions

$$
\begin{aligned}
\rho^{2} g_{R}(x-y) & =<\rho(x) \rho(y)>_{R}, \\
\rho^{2} \tilde{g}(x-y) & =<\rho(x) \rho(y)> \\
\rho^{2} g_{0}(x-y) & =<\rho(x)>_{\alpha}\left(<\rho(y)>_{R}\right)_{\alpha}, \\
\rho^{2} g_{1}(x-y) & =<\rho(x)>_{\alpha}<\rho(y)>_{\alpha},
\end{aligned}
$$

where we have assumed that the states of the system at a given temperature are in correspondence with the states at lower temperature. One imposes the constraint on the system at temperature $T$ that the function $g_{0}$ and $g_{1}$ are not zero. If $T=T_{R}$ this constraint can be easily satisfied. If $T<T_{R}$ this constraint forces the system at $T$ to stay out of equilibrium. It has been conjectured in [16 that the correlation functions in the metastable state (from which we can easily extract the energy) are given by $g_{1}(x)$ computed at $T_{R}=T_{M}(T)$.

\section{Solution of the equations}

Let us first discuss some technical points which are common to the soft and the hard sphere cases. In both cases the first task is to solve the replica symmetric HNC equation. For spherically symmetric functions in dimension three we use the Fourier transform for the radial dependance, in the following form:

$$
q \mathbf{h}(q)=2 \pi \int_{0}^{\infty} d r \sin (q r) r h(r) .
$$

We discretize this formula introducing in $r$ space a cutoff $R$ and a mesh size $a$. In this way we have a simple formula for the inverse Fourier transform and we can also use the fast Fourier transform algorithm. In most of the computations we have taken $a=1 / 32.5$ and $L=128 * a \approx 4$. We have tried smaller values of $a$ and larger values of $L$ without serious effects. The solution of the equations can be found either by using a library minimization program 2 , or a program which solves non linear equations. We have found first the solution

\footnotetext{
${ }^{2}$ When one has to minimize the free energy with respect to one variable and maximize it with respect to another one, we first minimize with respect to the first variable, later maximize with respect to the second, and iterate the procedure until convergence.
} 
at low enough density and then followed it by continuity while gradually increasing the density.

\subsection{The soft sphere case}

The HNC equation gives a description of the liquid phase which is not perfect, but precise enough for our purpose: The energy (or equivalently the pressure), does not depart more than $15 \%$ from the correct value (see fig.1), and the correlation function is also well reproduced (see fig. 2).

The numerical solution of the replicated HNC equations finds a dynamical transition at $\gamma \simeq 2.05$, and a static replica symmetry breaking solution at $\gamma \simeq 2.15$. In numerical simulations the glass transition is found at a smaller value of $\gamma$, namely $\gamma=1.6$. In the glass phase, the correlation function $g_{1}(r)$ is essentially a smoothed form of the function $\tilde{g}(r)$ plus an extra contribution at short distance which has integral near to 1 (see Fig.3). This form seems very reasonable: Considering the definition (3) of $g_{1}$, we see that it basically characterizes the average over $\alpha$ of the product $\rho_{\alpha}(x) \rho_{\alpha}(y)$, which is precisely expected to have this kind of peak structure.

In spite of this nice form for $g_{1}$, this solution has some problems. A first one is found on the value of the energy. The static energy as function of $\gamma$ (or equivalently of $T$ at density 1). is plotted in fig.1. Although there is a discontinuity in the specific heat, it is extremely small and the final effects on the internal energy are more or less invisible. The specific heat remains extremely large. Moreover the value of $m$ has a very unusual dependence on the temperature (see fig.4). In all the known models with one step replica symmetry breaking, the breakpoint $m$ varies linearly with $T$ at low temperatures. Here we have a very different behaviour. We have also computed the dynamical internal energy and found out that it differs from the equilibrium one by an extremely small account.

We conclude that if we consider the qualitative behavior of the correlation functions, we find a reasonable form, on the other hand the energy in the glassy phase turns out to be quite wrong.

\subsection{The hard sphere case}

The situation is quite similar in the case of hard sphere. The HNC approximation works reasonably in the liquid phase. The pressure, which can be extracted either from the free energy or from its relation with $g\left(1^{+}\right)$, does not depart from the correct one by more than

$15 \%$ in the liquid phase. The main defect is the absence of the peak around $r=\sqrt{3}$ and a too large value of the peak at $r=2$. The pressure seems to diverge proportionally to $\left(\rho_{c}-\rho\right)^{-2}$, where $\rho_{c}$ is around 1.6 , while the maximum possible density, corresponding to the fcc lattice, is $\sqrt{2}$.

Here replica symmetry is broken around $\rho \simeq 1.19$ and the dynamical transition is located at $\rho \simeq 1.17$. These values are very close to the result of the numerical simulations which find a freezing transition around $\rho \simeq 1.15$. Unfortunately also in this case the computed value for the pressure differs by a very small amount from the replica symmetric one and it is therefore unacceptable. Similar conclusions have also been reached for the Lennard Jones potential. 


\section{Conclusions}

The simple implementation of the replica approach to glasses which we have proposed here provides some interesting results, like the existence of a glass transition at a reasonable value of the density. However it is not satisfactory, in the sense that the effects of the transition on the thermodynamic of the systems are much too small.

At the moment we do not have a clear understanding of the reasons of this failure to grasp the thermodynamic properties of the glassy phase. Two possible explanations came to our mind. The first one would be that an approximation like that of HNC may miss some of the main physical characteristics of the problem in the glassy phase. A first look at the corrections to the free energy indicates that they are quite large, lending some support to this hypothesis. More work is needed to decide on what class of diagrams should be added to cure this problem. On the other hand we must also admit that we have not found so far a full derivation of the replicated HNC equations. A more proper approach would be to work with a free energy expressed as a functional of the density $\rho(x)$, seeking all the (non translational invariant) solutions corresponding to glass phases. Then the correlations and thermodynamics could be studied by giving to each solution a weight proportional to $\exp \left(-\beta F_{\alpha}\right), F_{\alpha}$ being the free energy of the solution labeled by $\alpha$.

We think that finding an analytic approximation scheme which produces reasonable results in the glass phase is within reach. The method we propose seems to be promising in this respect and shows how the replica method could be used to study the glass phase.

\section{Aknowledgements}

We thank D. Dean and R. Monasson for useful discussions.

\section{Figure Captions}

Fig.1 The static energy of a system of soft spheres as a function of the dimensionless inverse density $\gamma$ from numerical simulation (squares), from the replica symmetric HNC equation (full line) and from one step replica symmetry broken HNC equation (open circles).

Fig.2 The correlation function $\tilde{g}(r)$ of a system of soft spheres as a function of the distance at the dimensionless density $\gamma=1.6$ corresponding to the numerical glassy transition: numerical simulations (points) and replica symmetric HNC equation (full line).

Fig.3 The correlations $\tilde{g}(r)$ (full line) and $g_{1}(r)$ (broken line) obtained from the replicated hnc equations, as functions of the distance, for soft spheres at the density where replica symmetry is broken $(\gamma=2.15)$.

Fig. 4 The value of the breakpoint $m$ in the replica symmetry breaking solution for soft spheres as a function of the dimensionless density $\gamma$.

\section{References}

[1] For a recent introduction, see the papers in Science, 267 (1995) 1924, and the lectures in Liquids, freezing and glass transition, Les Houches 1989, JP Hansen, D. Levesque, J. Zinn-Justin Editors, North Holland. 
[2] T. R. Kirkpatrick and D. Thirumalai, Phys. Rev. Lett. 58, 2091 (1987); Phys. Rev. B36, 5388 (1987), J. Phys. A22 L149(1989).

[3] T. R. Kirkpatrick, D. Thirumalai and P.G. Wolynes, Phys. Rev. A40, 1045 (1989)

[4] G.Parisi, Slow dynamics in glasses, cond-mat 941115 and 9412034.

[5] S. Franz and J. Hertz; Phys. Rev. Lett. 74 (1995) 2114.

[6] J.P.Bouchaud, L.Cugliandolo, J. Kurchan and M.Mézard, "Mode coupling approximations, glass theory and disordered systems", preprint condmat 9511042, to appear in Physica A.

[7] J.-P. Bouchaud and M. Mézard; J. Physique I (France) 4 (1994) 1109.

[8] E. Marinari, G. Parisi and F. Ritort; J. Phys. A27 (1994) 7615; J. Phys. A27 (1994) 7647.

[9] L. F. Cugliandolo, J. Kurchan, G. Parisi and F.Ritort; Phys. Rev. Lett. 74 (1995) 1012.

[10] P. Chandra, L. Ioffe and D. Sherrington; cond-mat 9502018. P. Chandra, M. Feigelmann and L. Ioffe; preprint cond-mat 9509022.

[11] G. Parisi, , D-dimensional Arrays of Josephson Junctions, Spin Glasses and qdeformed Harmonic Oscillators, cond-mat preprint (1994).

[12] E. Marinari, G. Parisi and F. Ritort, Fully Frustrated Ising Spin Model on the Hypercube is Glassy and Aging, cond-mat preprint (1994).

[13] L.F. Cugliandolo, J. Kurchan, R. Monasson and G. Parisi, A mean field hard-spheres model of glass, J. Phys. (in press).

[14] A. Crisanti, H. Horner and H.-J. Sommers, Z. Phys. B92 257 (1993).

[15] L. F. Cugliandolo and J.Kurchan, Phys. Rev. Lett. 71, 1 (1993).

[16] S. Franz and G. Parisi, J.Phys. I (France) 5 (1995) 1401.

[17] R. Monasson, Phys.Rev.Lett. 75 (1995) 2847.

[18] A.Barrat, R.Burioni and M.Mézard, "Dynamics within metastable states in simple spin glass systems", preprint cond-mat/9511089.

[19] Y. Hiwatari, J. Phys. C13 (1980) 5899.

[20] M. Mézard, G. Parisi and M. A. Virasoro, Spin Glass Theory and Beyond, World Scientific, (Singapore 1987).

[21] See for instance J.P. Hansen and I.R. Macdonald, "Theory of simple liquids", (Academic, London, 1986), or H.N.V. Temperley, J.S. Rowlinson and G.S. Rushbrooke, "Physics of simple liquids", NorthHolland (Amsterdam 1968).

[22] T.V.Ramakrishnan and M.Yussouf, Phys.Rev. B19 2775(1979). 


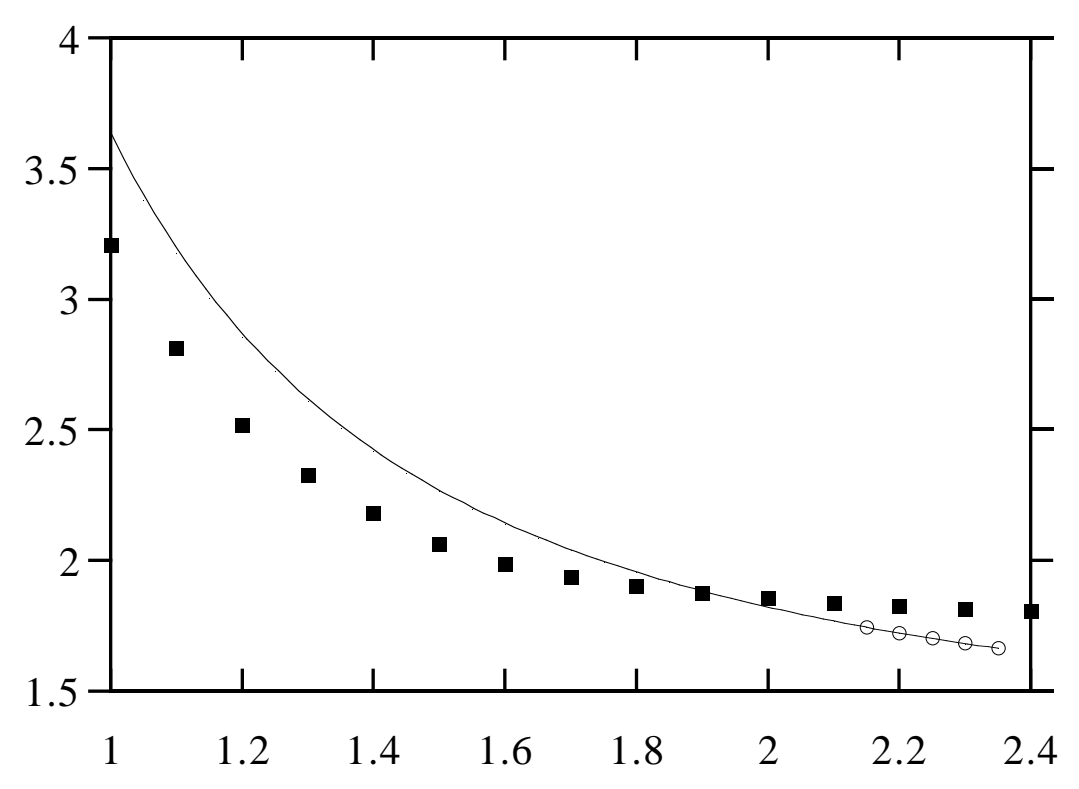


W 


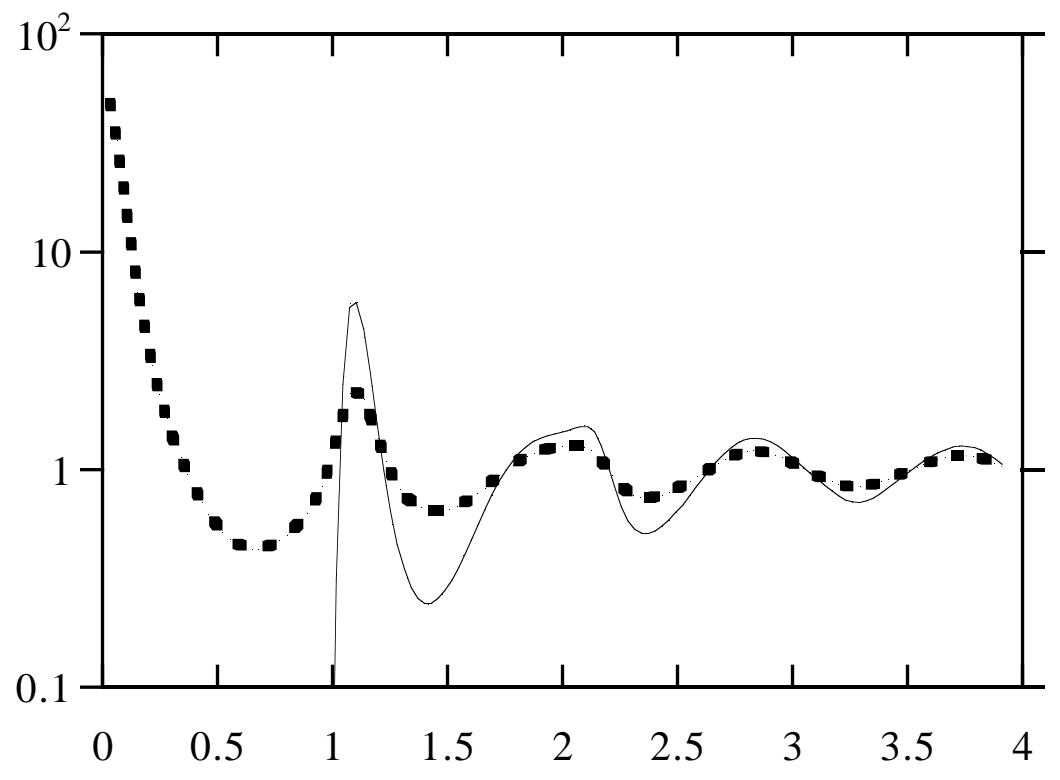




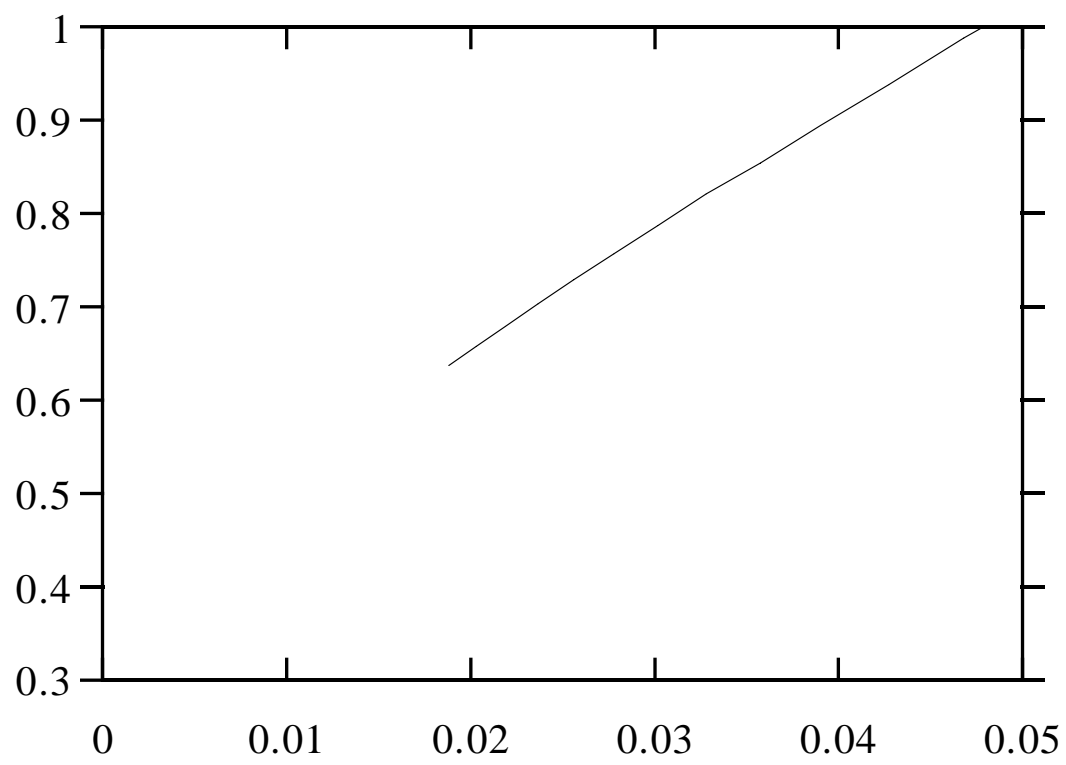

